Elsevier required licence: (C) 2019. This manuscript version is made available under the CC-BY-NC-ND 4.0 license $h$ ttp://creativecommons.org/licenses/by-nc-nd/4.0/

The definitive publisher version is available online at $h$ ttps://doi.org/10.1016/j.apenergy.2019.01.238 


\title{
Coordinated Operation of Electric Vehicle Charging and Wind Power Generation as a Virtual Power Plant: A Multi-stage Risk Constrained Approach
}

\author{
Mohammad Hossein Abbasi ${ }^{\mathrm{a}}$, Mehrdad Taki ${ }^{\mathrm{a}}$, Amin Rajabi ${ }^{\mathrm{b}}, \mathrm{Li} \mathrm{Li}^{\mathrm{b}}$, Jiangfeng Zhang ${ }^{\mathrm{b}}$ \\ ${ }^{a}$ The Department of Electrical Engineering, University of Qom, Qom, Iran. \\ ${ }^{b}$ The Faculty of Engineering and Information Technology, University of Technology Sydney, PO Box 123, Broadway, NSW 2007, Australia.
}

\begin{abstract}
As the number of electric vehicles (EVs) is steadily increasing, their aggregation can offer significant storage to improve the electric system operation in many aspects. To this end, a comprehensive stochastic optimization framework is proposed in this paper for the joint operation of a fleet of EVs with a wind power producer (WPP) in a three-settlement pool-based market. An aggregator procures enough energy for the EVs based on their daily driving patterns, and schedules the stored energy to counterbalance WPP fluctuations. Different sources of uncertainty including the market prices and WPP generation are modeled through proper scenarios, and the risk is hedged by adding a risk measure to the formulation. To obtain more accurate results, the battery degradation costs are also included in the problem formulation. A detailed case study is presented based on the Iberian electricity market data as well as the technical information of three different types of EVs. The proposed approach is benchmarked against the disjoint operation of EVs and WPP. Numerical simulations demonstrate that the proposed strategy can effectively benefit EV owners and WPP by reducing the energy costs and increasing the profits.
\end{abstract}

Keywords: Electric vehicle, Wind power producer, Wind power prediction, Virtual power plant, Conditional value-at-risk, Stochastic programming.

\section{Introduction}

Investment in renewable energy has been increasing steadily in recent years, and as a result, renewable based generation, especially wind power production, has significantly expanded its market share. The advantages of wind generation for decreasing the operational costs and reducing the emissions of hazardous gases are well known. However, the intermittent nature and inherent uncertainty of wind generation can jeopardize power system's reliable operation, which makes it difficult for wind power producers (WPPs) to compete with large conventional power plants in electricity markets. Various methods have been proposed in the literature to overcome these problems, which includes mainly the following: 1) using feed-in tariffs as a mechanism to support WPPs, which guarantees a minimum tariff irrespective of electricity market prices $[1,2], 2)$ using storage facilities that can store the excess energy of wind generation during off-peak hours and feed electricity back to the grid during peak hours, in particular battery storage systems [3, 4], plug-in electric vehicles (EVs) [5, 6], and 3) other technologies like compressed air energy storages or pumped-storage units [7,8], joint operation with demand response (DR) providers to utilize the flexibility of time-shiftable loads $[9,10]$, and participating in various market structures to assist WPPs in competing with other producers [11].

EVs are viewed as a promising technology which are becoming increasingly popular in the global market. From the power system perspective, they introduce new challenges as their uncontrolled charging may result in increased reserve requirements, increased electricity generation, probable network reinforcement, and higher market prices [12]. However, their ability in storing electricity and supplying it back to the network in the vehicle-to-grid (V2G) mode positions them as mobile storage systems that provide distributed storage to power grids. In this regard, they are

Email addresses: m.taki@qom.ac.ir (Mehrdad Taki), li.li@uts.edu.au (Li Li) 


\begin{tabular}{|c|c|c|c|}
\hline \multicolumn{4}{|c|}{ Nomenclature } \\
\hline \multicolumn{2}{|c|}{ Indices and Sets: } & \multirow{2}{*}{$\begin{array}{l}P^{\mathrm{W}, \max } \\
P_{t \omega}^{\mathrm{W}}\end{array}$} & \multirow{2}{*}{$\begin{array}{l}\text { Wind farm capacity } \\
\text { Wind power generation }\end{array}$} \\
\hline$\omega \in \Omega$ & Scenarios & & \\
\hline$t \in T$ & Time slots & $P_{t \omega}$ & Total scheduled power \\
\hline \multirow[t]{2}{*}{$v \in \mathcal{V}$} & Vehicles & $r_{t \omega}^{+}$ & Positive imbalance ratio \\
\hline & & $r_{t \omega}^{-}$ & Negative imbalance ratio \\
\hline \multicolumn{4}{|c|}{ Abbreviations and Superscript Symbols: } \\
\hline,+- & Positive/negative deviations, charge/dis- & \multicolumn{2}{|c|}{ EV Parameters: } \\
\hline & charge & $\delta_{v}$ & Initial battery state of charge \\
\hline$S O C$ & State-of-charge & $\kappa_{v}^{+}, \kappa_{v}^{-}$ & Grid to vehicle efficiency and vice versa \\
\hline$w p p$ & Wind power producer & $v_{t \omega v}$ & $\begin{array}{l}\text { Binary auxiliary variable. It equals } 1 \text { if } \\
\text { vehicle is available }\end{array}$ \\
\hline \multicolumn{2}{|c|}{ Market Parameters: } & $\rho_{t \omega v}$ & Battery loss of charge from driving \\
\hline$\Delta_{t \omega}^{+}$ & Positive imbalance power & M & Sufficiently large constant \\
\hline$\Delta_{t \omega}^{-}$ & Negative imbalance power & $\theta_{t \omega v}$ & Binary auxiliary variable \\
\hline$\Delta_{t \omega}$ & Imbalance power & $\varrho_{t \omega v}^{+}, \varrho_{t \omega v}^{-}$ & Vehicle charge/discharge \\
\hline$\ell$ & Wind power adjustment offer bound & $\varrho_{t \omega v}^{S O C}$ & Battery state of charge \\
\hline$\eta$ & Demand response upper bound & $\vartheta_{v}^{\max }$ & Battery capacity \\
\hline$\lambda_{t \omega}^{\mathrm{A}}$ & Adjustment market price & $\vartheta_{v}^{\min }$ & Minimum battery charge \\
\hline$\lambda_{t \omega}^{\mathrm{D}}$ & Day-ahead market price & $C_{t \omega v}^{\mathrm{EV}}$ & Battery degradation cost \\
\hline$\pi_{\omega}$ & Probability of scenario & $E_{v}^{\max }$ & Maximum rate of charge/discharge \\
\hline$D_{t \omega}^{\mathrm{A}}$ & Demand side adjustment bid & & \\
\hline$D_{t \omega}^{\mathrm{D}}$ & Demand side day-ahead bid & \multicolumn{2}{|c|}{ Risk Measure: } \\
\hline$L_{t \omega}^{\mathrm{A}}$ & Demand side adjustment offer & $\alpha$ & Confidence level \\
\hline$L_{t \omega}^{\mathrm{D}}$ & Demand side day-ahead offer & $\beta$ & Weighting parameter \\
\hline$L_{t \omega}$ & Demand side scheduled power & $\xi_{\omega}$ & Scenario-specific auxiliary variable \\
\hline$P_{t \omega}^{\mathrm{A}}$ & Scheduled adjustment offer & $\zeta$ & Continuous auxiliary variable \\
\hline$P_{t \omega}^{\mathrm{D}}$ & Scheduled day-ahead offer & & \\
\hline
\end{tabular}

considered as a suitable means to stabilise the network and facilitate the integration of renewable resources [13]. Furthermore, in comparison with other storage technologies, they do not represent an upfront capital cost for grid operators and power suppliers. However, integration of EVs as storage into the network leads to battery degradation cost which is expressed by battery capital cost and depth-of-discharge (DoD). Usually, an "aggregator" coordinates charging of an EV fleet. The presence of this new entity is necessary for managing the vehicles' charging, either directly or indirectly, and seizing the potential of EVs [6]. Specially, aggregators can benefit EV owners by participating in different electricity markets on behalf of individual vehicles. They can also join a virtual power plant (VPP) which is usually defined as a coalition composed of (dispatchable and non-dispatchable) power producers, energy storage systems, and demand response providers.

In this paper we target the aforementioned problems of WPPs by a hybrid approach, which consists of the con- 
struction of a VPP based on the coordinated operation of a relatively large EV fleet with the WPP; and also the participation of this VPP in a three-settlement pool-based market to achieve the maximum profit. The joint operation of WPP with time-shiftable loads has been addressed in various studies [9, 10, 14]. Recently, some studies have also highlighted the coordinated operation of WPPs with EVs. Unlike the flexible loads that mostly operate by changing their electric usage in response to certain signals [15], EVs have the ability of storing energy and shifting their energy usage. These features are especially important from two perspectives: 1) EVs can assist a WPP to manage the unpredictability in its production and maximize its profits by storing the excess power of WPP in their own batteries and selling it to the grid when needed, and 2) for decreasing the electricity costs for the EV owners. The results of our study show that the WPP can achieve higher profits in the VPP operation, whereas significant reductions in electricity costs can be attained for vehicle owners.

\subsection{Literature Review}

\subsubsection{EV aggregation}

In recent years, a great deal of research has explored possible effects of integration of EVs in electrical networks. Technical aspects of EV integration such as the impacts on distribution system, the need for network reinforcement, and the proper allocation of charging facilities have been analysed in some studies. The operation and management of EVs have been highlighted in a wider range of publications which mainly study the aggregation of EVs, the interactions with electricity markets, and the coordinated operation of EVs with renewable energy resources.

Aggregation of EVs is addressed in different publications, studying their operation in various market settlements. Ref. [16] develops a methodology for an aggregator that optimizes charging and discharging of EVs by modelling driving patterns and forecasting market prices. Furthermore, it considers the cases when the aggregator is a pricetaker or a price-maker. Ref. [17] models an EV aggregator who satisfies the demand of EV owners and participates in frequency regulation to maximize its own benefit. The proposed framework includes several modules allowing the aggregator to track the load frequency control signal and to schedule the EVs. The authors in [18] formulate the bidding strategy of an aggregator as a bi-level problem. The lower level problem addresses the market clearing, while the upper level one considers the charging cost minimization of the aggregator. The aggregate charging power is optimised by using the dynamic programming algorithm. Ref. [19] approaches the scheduling of EV charging in coordination with the system operator. By considering a very large number of EVs, this paper studies the necessary adoptions that are needed in DA markets to introduce EV aggregators. In a recent work [20], a novel inter-aggregator agent is introduced which acts as a third-party coordinator of several EV aggregators and bids on behalf of them in the markets. Then a framework is proposed for distributing the energy costs among these aggregators.

In terms of EV fleet coordination, several approaches have been proposed which can be generally categorized as centralized and decentralized approaches [21]. In centralized algorithms, a global optimizer communicates with all devices and schedules the energy usage of each device [22]. This approach can guarantee optimal solutions. In decentralized approaches, also known as agent-based methods, each device makes autonomous decisions and adjusts this schedule until a global equilibrium is reached. In [23], a decentralized algorithm is used for real-time EV charging control. Control signals are distributed to the charging stations where they perform optimization locally regarding the EV connected to them.

There are various sources of uncertainty in EV scheduling optimization problems: stochastic arrival times, unknown departure times, inaccurate battery requirements, uncertain market prices, etc. Different approaches have been used to model these uncertainties. For instance, [24] develops a stochastic optimization strategy to take into account the uncertainty in arrival and departure times of EVs. Compared with the deterministic approach, the proposed algorithm reduces the charging costs of aggregator at peak hours. A Monte-Carlo simulation is applied in [25] to model uncertainties in transportation patterns and a rolling horizon approach is presented in [21] which accounts for uncertainties. In [22] a set of scenarios are defined to model different uncertainties and conditional-value-at-risk (CVaR) measure is added to the problem formulation. Other papers also use robust optimization for modelling uncertainties $[26,27]$. In [26], DA market prices are modelled through a set of scenarios and the uncertainties in driving requirements are modelled using the confidence bounds. Ref. [27] uses a robust optimization framework to model wholesale electricity prices and the corresponding optimal charging policy.

Extra costs are imposed on EV owners due to degradation of batteries when they are used as storages in aggregation process. While some publications studied the technical aspects of battery degradation in detail [28], others have 
incorporated battery degradation costs in the optimization problems [29, 30]. Ref. [29] proposes an EV aggregator operation mechanism considering battery degradation influence. It assumes that both charging and discharging cycles can lead to battery degradation and obtains the costs by considering the capital cost of battery, battery capacity, cycling times, and depth of discharge. Authors in [30] introduce a bidding strategy to maximize the profits of an EV aggregator that participates in energy and reserve markets while compensating EV owners for the degradation of batteries. The battery degradation cost is formulated using a more complicated model.

\subsubsection{Joint operation of $E V s$ and WPP}

The operation of WPPs in electricity markets is investigated in various studies. The difference among these studies mainly relates to the market settlements, bidding in the markets either as a price-taker or a price-maker, and considering the competition among rival wind power producers. In this regard, offering strategies of a WPP in dayahead (DA), adjustment, and balancing markets are formulated in [31]. Ref. [32] proposes the participation of a WPP in day-ahead balancing, and regulation markets. The WPP is considered to be a price-maker in both DA and balancing markets. On the other hand, [1] and [33] address the problem of a WPP that bids in DA and balancing markets. While the former considers the WPP as a price-maker in the balancing market, the latter deals with it as a price-maker in the DA market. Moreover, the rivals' offers are taken into account in [2] by modelling them as a set of scenarios. Finally, in [34], WPPs share their wind power forecasts with others to improve their profit through making more informed decisions.

Furthermore, the joint operation of WPPs and EV aggregators are presented in a few studies. Ref. [35] investigates the best options for a WPP who offers in the balancing and regulation markets under three different scenarios including the operation of WPP only, WPP with storage systems, and WPP with EVs. The results demonstrate the advantages of using EVs over the other cases. The model in [36] minimizes the expected grid operation cost in a security constrained unit commitment problem which includes the coordinated integration of aggregated EVs and WPPs. A dispatch model is also introduced in [37] which takes into account EVs and wind power, and solves the problem using a particle swarm optimization. In [6] the flexibility of the aggregated EV fleet is represented by a probabilistic battery model. The aggregator purchases energy from the DA market and offers balancing services for the WPP. The authors in [38] present a unit commitment and economic dispatch model to incorporate wind generators. The proposed model utilises the flexibility of EVs to compensate for the wind generation uncertainty. Ref. [39] develops a power plant model of EVs based on the travelling behaviours and determines the available capacity of EV aggregation. Afterwards, an active power regulation strategy is proposed to offset the imbalance power caused by stochastic wind farms.

\subsection{Contributions}

This paper presents a comprehensive model for the coordinated operation of an EV aggregator with a WPP. In summary, the contributions of this paper are as follows: 1) Compared with existing studies, the proposed model considers the detailed constraints of WPP and each EV, and determines the charging and discharging schedules of EVs for each hour in a day-ahead scheme. Furthermore, battery degradation cost is included in the problem formulation. 2) To model various interactions of aggregator and WPP in the markets, a more comprehensive market structure is formulated. In comparison to most of previous studies, here a three-settlement pool-based market including DA, intra-day (adjustment), and balancing markets is formulated. Also, by adding the CVaR risk measure, the problem is considered to be a risk-averse optimization. 3) The case study differs from previous works as it not only analyses a large number of EVs (1000), but also comprises three different kinds of vehicles from three manufacturers. It offers various advantages including the analysis of scheduling of EVs with different characteristics. 4) A fuzzy-based method is developed for the wind power forecast of WPP.

\subsection{Paper Organization}

The remaining sections of the paper are structured as follows. Section 2 explains the underlying assumptions including the market framework, aggregator problem, and forecast models for the WPP and market prices. Section 3 introduces the mathematical formulation of proposed method. In Section 4 the benchmark disjoint operation of WPP and DR is formulated. The details of an empirical evaluation through a case study are reported in Section 5. Finally, conclusions are presented in Section 6. 


\section{Problem Description}

This paper considers the jointly optimized operation of a WPP and a DR aggregator (DRA) as a VPP. DRA aggregates a group of EVs, meets their energy requirements, and offers the stored energy in EVs' batteries to the market. By the proposed optimization framework, DRA purchases energy at off-peak periods with low prices and sells the excess energy at peak periods. Accordingly, the cost of EVs' energy consumption can be considerably reduced. This proposed configuration is compared with another situation in which DRA and WPP operate independently. The case studies demonstrate that the joint operation is more economically beneficial. In the following, the market framework is explained first, then the problems of producer and demand side are illustrated, and finally, different sources of uncertainty are described and modelled.

\subsection{Market Framework}

A three-settlement pool-based market including DA, adjustment, and balancing markets is considered in this study. DA is the main market for energy trading. Producers and consumers send their offers and bids to the market operator one day prior, and the market operator clears the market. Adjustment markets are similar to the DA market, however, they are cleared closer to the time of actual energy delivery. Therefore, they are useful for addressing producers' uncertainty. Balancing market mechanism allows producers to rectify their imbalances. The main energy transactions accomplish in DA and adjustment markets, and balancing market is used just to cover the deviations. So, balancing market does not have an independent price, and its price is a factor of DA price.

\subsection{Producer and Demand Side}

As mentioned in the previous section, the intermittent nature of wind power generation is a main source of uncertainty in electricity markets. Management of EVs, as time-shiftable loads, by DRA and the joint operation of WPP with DRA can compensate some uncertainties of WPP. During off-peak hours, EVs can store the excess production of WPP, and sell electricity to the grid during peak hours. In this manner, DRA purchases the off-peak energy which may be produced by WPP at low costs to charge batteries of its clients. DRA may even buy more energy than its real requirement and stores it in EVs' batteries in order to sell it in peak periods. This is achievable since the majority of vehicles are in the parking position most of the time [40]. This will be explained in more detail in the next sections. This coordination between WPP and DRA has benefits for both sides. In more detail, the operation of VPP can be summarized as follows:

1. DRA tries to procure the required energy for its clients at the periods with low prices. Moreover, DRA may purchases more energy, in order to sell the additional energy in peak periods and compensate its cost.

2. VPP optimizes the surplus energy that DRA has bought, along with the produced energy of WPP.

3. The joint operation of DRA and WPP as a VPP results in more profit, compared with the disjoint operation.

\subsection{Uncertainty Modeling Using Scenarios}

Changes in generation and demand in electricity grids lead to the price uncertainty. In addition, the intermittent nature of wind speed and direction is the origin of uncertainty in wind farm production. These uncertain variables can be modeled through stochastic processes. In this paper, scenarios that model markets prices/coefficients are generated using ARMA models, and scenarios of wind power production are generated through an Adaptive Neuro-Fuzzy Inference System (ANFIS).

ARMA models are one of the most well-known methods for scenario generation, which are used in various papers on electricity markets with stochastic programming problems to estimate future prices. For instance, ARMA is used in $[41,42]$ to model market prices.

The general formulation of ARMA which is used for the estimation of price, $\lambda_{t}$, is as follows:

$$
\left(1-\sum_{j=1}^{N_{P}} \phi_{j} B^{j}\right)\left(1-\sum_{j=1}^{N_{P_{S}}} \Phi_{j} B^{j S}\right) \lambda_{t}=\left(1-\sum_{j=1}^{N_{Q}} \psi_{j} B^{j}\right)\left(1-\sum_{j=1}^{N_{Q s}} \Psi_{j} B^{j S}\right) \varepsilon_{t},
$$

where $\phi_{j}, j \in\left\{1, \cdots, N_{P}\right\}$, represent auto regressive, $\Phi_{j}, j \in\left\{1, \cdots, N_{P s}\right\}$, show seasonal auto regressive, $\psi_{j}, j \in$ $\left\{1, \cdots, N_{Q}\right\}$, are moving average, and $\Psi_{j}, j \in\left\{1, \cdots, N_{Q s}\right\}$, indicate seasonal moving average parameters. $S$ represents seasonality order, $B^{\mathrm{X}}$ is back shift operator, and $\varepsilon_{t}$ stands for white noise. 


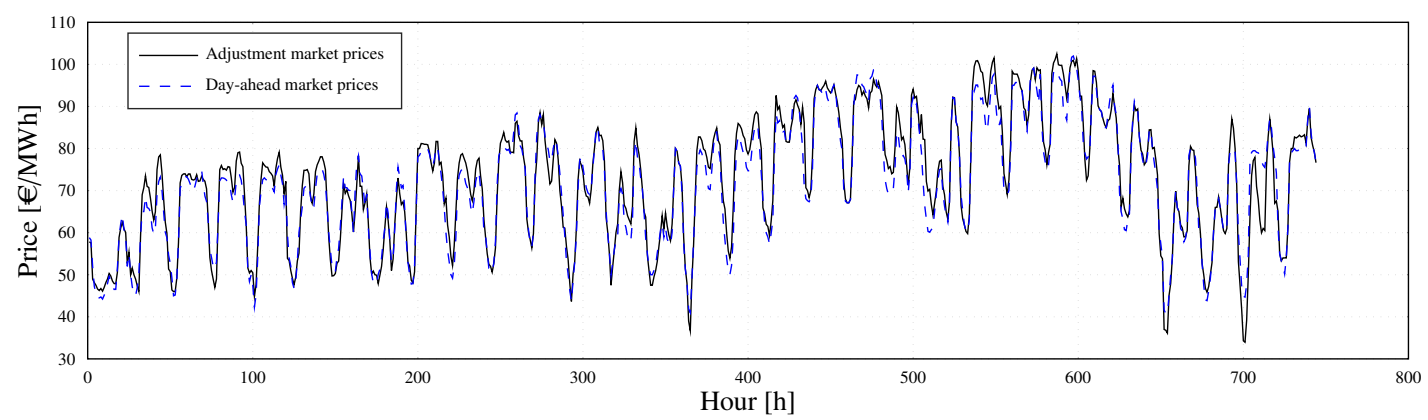

Figure 1: Spanish day-ahead and adjustment markets prices for January 2017

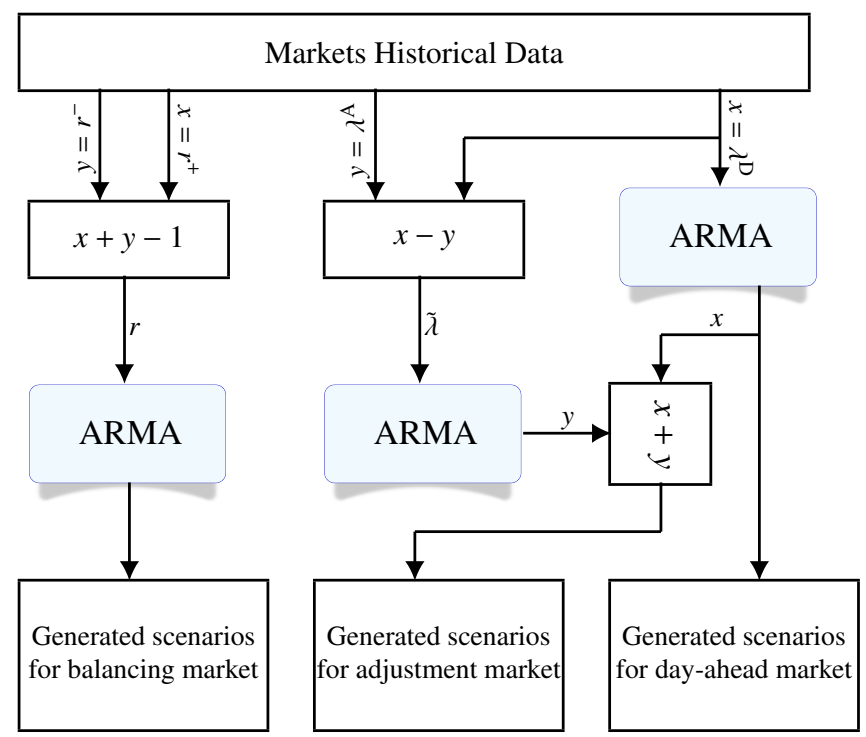

Figure 2: Scenario generation for markets prices using ARMA

ARMA coefficients in (1) are inferred by historical data. After calculation of coefficients, the future prices are estimated.

Since DA market clears before the other markets, it is assumed that its price, $\lambda^{\mathrm{D}}$, is not correlated to adjustment price. Therefore, scenarios of DA price are generated independently.

After the generation of DA price scenarios, since adjustment prices are correlated with DA prices (Fig. 1), they are modeled as DA prices, plus a noise factor, $\tilde{\lambda}$, and scenarios are generated for $\lambda^{\mathrm{A}}$.

$$
\lambda^{\mathrm{A}}=\lambda^{\mathrm{D}}+\tilde{\lambda}
$$

Balancing price is modeled as in (3), where $\lambda^{\mathrm{B}}$ is the balancing price, and $r=r^{+}+r^{-}-1$ is the imbalance coefficient [43].

$$
\begin{gathered}
\lambda^{\mathrm{B}}=r \cdot \lambda^{\mathrm{D}}, \\
r= \begin{cases}r^{+} & \text {if } r^{+} \neq 1 \\
r^{-} & \text {if } r^{+}=1 .\end{cases}
\end{gathered}
$$

Note that $r^{+} \leq 1$ and $r^{-} \geq 1$. The process of scenario generation for market prices is depicted in Fig. 2 . 


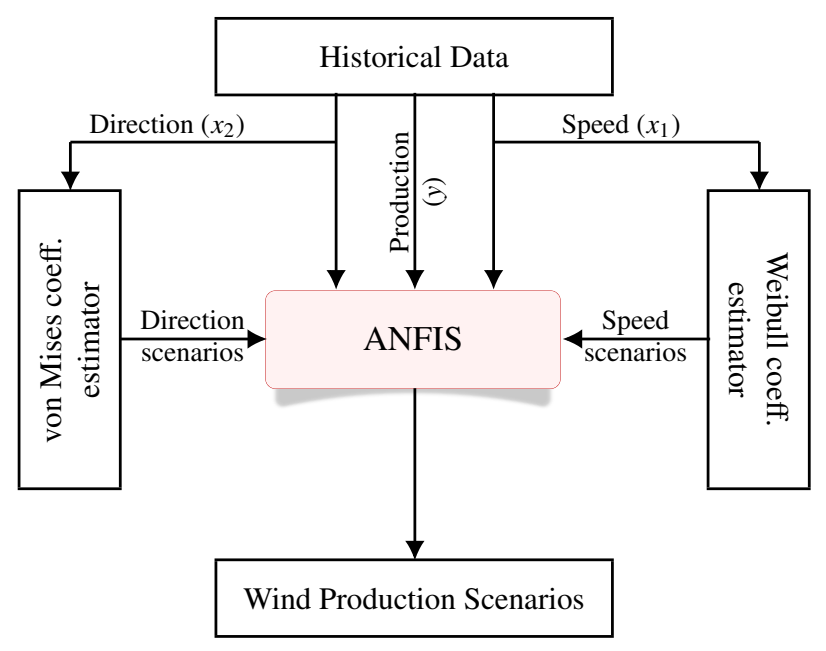

Figure 3: Scenario generation for wind farm production using ANFIS

Scenario generation of WPP production is perfomed using the ANFIS, which estimates an emulating model of wind farm [44]. The ANFIS inputs are wind speed and direction with Weibull and von Mises distributions, respectively. Using these distributions, scenarios for the inputs of ANFIS are generated. Finally, ANFIS generates the scenarios for WPP production.

The structure of ANFIS system is shown in Fig. 3. Historical data includes three data sets. The first two data sets consist of the data of wind speed and direction. The other data set contains the historical data of wind farm production. The considered fuzzy system is Takagi-Sugeno-Kang (TSK) [45] in which Fuzzy C-Means (FCM) algorithm is used in order to identify the parameters of the model. Gaussian membership functions are considered for each input, and the system structure comprises three fuzzy logic if-then rules.

\section{Problem Formulation}

The goal of the problem is to simultaneously minimize the cost of the consumer and maximize the profit of the producer. The consumer is the DRA which aggregates the total EVs' demand and determines the vehicles' charging time and charging duration. The producer is a VPP managing the cooperation between DRA and WPP.

It is expected that vehicles are charged at the inexpensive (or off-peak) periods and discharged at the peak periods. The periods that vehicles are unavailable should be declared to DRA by EV owners before the DA market price is cleared, and the DRA will also be able to monitor and forecast EV usage through surveys and historical EV usage data (see [46]).

\subsection{Electric Vehicle Constraints}

The considered vehicle constraints are as follows:

$$
\begin{array}{ll}
\forall t, \omega, v: & \varrho_{t \omega v}^{+}+\varrho_{t \omega v}^{-} \leq v_{t \omega v} E_{v}^{\max }, \\
\forall t, \omega, v: & \varrho_{t \omega v}^{S O C}=\varrho_{(t-1) \omega v}^{S C}+\varrho_{t \omega v}^{+} \kappa_{v}^{+}-\frac{\varrho_{t \omega v}^{-}}{\kappa_{v}^{-}}-\rho_{t \omega v}, \\
\forall t, \omega, v: & \vartheta_{v}^{\min } \leq \varrho_{t \omega v}^{S O C} \leq \vartheta_{v}^{\max }, \\
\forall t, \omega, v: & \rho_{t \omega v} \leq \mathrm{M}\left(1-v_{t \omega v}\right), \\
\forall t, \omega, v: & 0 \leq \varrho_{t \omega v}^{+} \leq \mathrm{M} \theta_{t \omega v}, \\
\forall t, \omega, v: & 0 \leq \varrho_{t \omega v}^{-} \leq \mathrm{M}\left(1-\theta_{t \omega v}\right),
\end{array}
$$




$$
\forall \omega, v: \quad 0 \leq \varrho_{0, \omega v}^{S O C}=\delta_{v}
$$

Constraints (5) are batteries charging and discharging limitations, because in the considered time slots a battery has some maximum limits for charging and discharging. Constraints (6) impose that $\varrho_{t \omega v}^{S O C}$ in every time slot and scenario (at the end of the considered time slot) is equal to the battery's state of charge (SOC) at the end of the previous time slot plus/minus possible amount being charged/discharged, and minus battery loss of charge from driving. Constraints (7) are batteries' SOC limitations. Constraints (8) imply that when a vehicle is moving, it is unplugged from the network. Constraints (9) and (10) differentiate between vehicles charging and discharging. Constraints (11) describe the initial values of batteries SOC.

\subsection{Proposed Formulation}

The following proposed formulation for the joint operation of WPP and DRP as a VPP is a three-stage stochastic programming problem for bidding DRA consumption and offering VPP sell. The first, second and third stages are decision makings for day-ahead, adjustment, and balancing markets, respectively. Therefore, the considered variables for trading energy in each of these markets are variables for the respective stage.

1. First stage variables: $P_{t, \omega}^{\mathrm{D}}, L_{t, \omega}^{\mathrm{D}}$, and $D_{t, \omega}^{\mathrm{D}}$

2. Second stage variables: $P_{t, \omega}^{\mathrm{A}}, L_{t, \omega}^{\mathrm{A}}$, and $D_{t, \omega}^{\mathrm{A}}$

3. Third stage variables: $\Delta_{t, \omega}^{+}$and $\Delta_{t, \omega}^{-}$

It is assumed that the sampling time is one hour, and therefore, in the objective function, multiplication by time is omitted. The proposed formulation is:

Max. $\mathcal{E}=\sum_{\Omega} \pi_{\omega} \sum_{\mathcal{T}}\left[\lambda_{t \omega}^{\mathrm{D}}\left(P_{t \omega}^{\mathrm{D}}-D_{t \omega}^{\mathrm{D}}\right)+\lambda_{t \omega}^{\mathrm{A}}\left(P_{t \omega}^{\mathrm{A}}-D_{t \omega}^{\mathrm{A}}\right)+\lambda_{t \omega}^{\mathrm{D}}\left(r_{t \omega}^{+} \Delta_{t \omega}^{+}-r_{t \omega}^{-} \Delta_{t \omega}^{-}\right)-\Pi_{t \omega}\right]+\beta\left(\zeta-\frac{1}{1-\alpha} \sum_{\Omega} \pi_{\omega} \xi_{\omega}\right)$,

s.t.

$$
\text { (5)-(11), }
$$

$\forall t, \omega: \quad P_{t \omega}=P_{t \omega}^{\mathrm{D}}+P_{t \omega}^{\mathrm{A}}$,

$\forall t, \omega: \quad P_{t \omega} \leq P^{\mathrm{W}, \max }+\eta \sum_{\mathcal{V}} E_{v}^{\max }$,

$\forall t, \omega: \quad P_{t \omega}^{\mathrm{D}} \leq P^{\mathrm{W}, \max }+\eta \sum_{\mathcal{V}} E_{v}^{\max }$,

$\forall t, \omega: \quad \Delta_{t \omega}=P_{t \omega}^{\mathrm{W}}+L_{t \omega}-P_{t \omega}$,

$\forall t, \omega: \quad \Delta_{t \omega}=\Delta_{t \omega}^{+}-\Delta_{t \omega}^{-}$,

$\forall t, \omega: \quad \Delta_{t \omega}^{+} \leq P_{t \omega}^{\mathrm{W}}+L_{t \omega}$,

$\forall t, \omega: \quad \Delta_{t \omega}^{-} \leq P^{\mathrm{W}, \max }+\eta \sum_{\mathcal{V}} E_{v}^{\max }$,

$\forall t, \omega, \omega^{\prime}: \quad\left(P_{t \omega}^{\mathrm{D}}-P_{t \omega^{\prime}}^{\mathrm{D}}\right) \cdot\left(\lambda_{t \omega}^{\mathrm{D}}-\lambda_{t \omega^{\prime}}^{\mathrm{D}}\right) \geq 0$,

$\forall t, \omega, \omega^{\prime}: \quad P_{t \omega}^{\mathrm{D}}=P_{t \omega^{\prime}}^{\mathrm{D}}: \lambda_{t \omega}^{\mathrm{D}}=\lambda_{t \omega^{\prime}}^{\mathrm{D}}$,

$\forall t, \omega, \omega^{\prime}: \quad P_{t \omega}^{\mathrm{A}}=P_{t \omega^{\prime}}^{\mathrm{A}}: \lambda_{t \omega}^{\mathrm{A}}=\lambda_{t \omega^{\prime}}^{\mathrm{A}}$,

$\forall t, \omega: \quad-\ell \cdot P_{t \omega}^{\mathrm{D}} \leq P_{t \omega}^{\mathrm{A}} \leq \ell \cdot P_{t \omega}^{\mathrm{D}}$,

$\forall t, \omega: \quad L_{t \omega}=L_{t \omega}^{\mathrm{D}}+L_{t \omega}^{\mathrm{A}}$,

$\forall t, \omega: \quad L_{t \omega}^{\mathrm{D}} \leq \eta \sum_{\mathcal{V}} E_{v}^{\max }$,

$\forall t, \omega: \quad L_{t \omega}=\sum_{V} \varrho_{t \omega v}^{-}$, 


$$
\begin{aligned}
\forall t, \omega: & D_{t \omega}^{\mathrm{D}}+D_{t \omega}^{\mathrm{A}}=\sum_{\mathcal{V}} \varrho_{t \omega v}^{+}, \\
\forall t, \omega: & \Pi_{t \omega}=\sum_{\mathcal{V}} C_{t \omega v}^{\mathrm{EV}}, \\
\forall \omega: & \zeta-\sum_{\mathcal{T}}\left[\lambda_{t \omega}^{\mathrm{D}}\left(P_{t \omega}^{\mathrm{D}}-D_{t \omega}^{\mathrm{D}}\right)+\lambda_{t \omega}^{\mathrm{A}}\left(P_{t \omega}^{\mathrm{A}}-D_{t \omega}^{\mathrm{A}}\right)+\lambda_{t \omega}^{\mathrm{D}}\left(r_{t \omega}^{+} \Delta_{t \omega}^{+}-r_{t \omega}^{-} \Delta_{t \omega}^{-}\right)-\Pi_{t \omega}\right] \leq \xi_{\omega}, \\
\forall \omega: & \xi_{\omega} \geq 0 \\
\forall t, \omega: & P_{t \omega}, P_{t \omega}^{\mathrm{D}}, \Delta_{t \omega}^{+}, \Delta_{t \omega}^{-} \geq 0 \\
\forall t, \omega: & L_{t \omega}, L_{t \omega}^{\mathrm{D}}, L_{t \omega}^{\mathrm{A}}, D_{t \omega}^{\mathrm{D}}, D_{t \omega}^{\mathrm{A}} \geq 0 \\
\forall t, \omega, v: & \varrho_{t \omega v}^{+}, \varrho_{t \omega v}^{-}, \rho_{t \omega v}, \varrho_{t \omega v}^{S O C} \geq 0 \\
\forall t, \omega, v: & v_{t \omega v}, \theta_{t \omega v} \in\{0,1\} .
\end{aligned}
$$

The optimization problem (12) is composed of five terms. The first two terms show the expected revenue of VPP from selling energy to the DA and adjustment markets minus its expected cost from purchasing energy in these markets. The third part includes the revenue/cost of positive/negative energy deviation which is corrected in the balancing market. The next term represents the cost of battery degradation. The last part represents the CVaR risk criterion with weighting parameter $\beta$. Constraints (13) shows the total offered energy in DA and adjustment markets. Constraints (14) represents the upper limit of offered power. Maximum power that can be offered by VPP is equal to the wind farm capacity, i.e., $P^{\mathrm{W} \text {,max }}$ plus a fraction of batteries' discharge rate. Parameter ' $\eta$ ' is less than or equal to 1 and implies that at a certain time period, some of the vehicles may not be accessible since they are in the driving status. Note that the amount of DRA's power offer depends not only on the amount of power that it purchases at a specific time slot but also on batteries state of charge, which means that the DRA offer depends on previous time slots as well. It is worth mentioning that the amount of electricity that is saved inside the EVs' batteries could be more than $\sum_{v} E_{v}^{\max }$ but DRA would not be able to offer such amount of energy, since the offer is limited to the batteries' rate of discharge. Constraints (15) limit the VPP's offer in the DA market. These constraints are involved due to the fact that the adjustment offer can be negative. They prevent from a large positive DA offer and a large negative adjustment offer that satisfy Constraints (23) but are out of practical WPP capacity. Constraints (16) show the deviations. The total deviation is equal to the generated wind power plus the demand side offered power and minus the total offered power. Constraints (18) and (19) state the maximum possible amount of positive and negative power deviations, respectively. Constraints (20) impose non-decreasing offering curves for the DA energy offer. Constraints (21) and (22) impose non-anticipative condition for the DA and adjustment energy offer, respectively. Constraints (23) determine the lower and upper bounds of adjustment offer. Note that $P_{t \omega}^{\mathrm{A}}$ can be negative. Constraints (24)-(25) pertain to the demand side schedule. Constraints (26) show that the total demand side schedule in each time slot and scenario is equal to the total amount of discharged energy from EVs in that time slot and scenario. Constraints (27) is related to the amount of purchased energy from DA and adjustment markets which is equal to the EVs' charge. The cost of battery degradation is represented in constraints (28), in which $C_{t \omega v}^{\mathrm{EV}}=C_{v}^{\mathrm{bat}} / L_{t \omega v}^{\mathrm{DoD}}$, where $C_{v}^{\mathrm{bat}}$ is battery capital cost and $L_{t \omega v}^{\mathrm{DoD}}$ is battery lifetime in cycles [47]. Constraints (29) describes the CVaR risk constraints for the maximization problem. Finally, positive variables of the problem are shown by Constraints (30)-(33) and binary variables are represented by Constraints (34).

\section{Benchmark Scheme}

In this section, a benchmark scheme is presented to evaluate the efficiency of the proposed optimized formulation in the previous section. We consider a case in which WPP and DRA participate independently in the market. In the considered scheme, WPP tries to maximize its profit by participating in a three-settlement market, and also taking the CVaR risk criterion into account. This optimization problem [9], is illustrated by (35):

Max. $\quad \mathcal{E}^{\mathrm{WPP}}=\sum_{\Omega} \pi_{\omega} \sum_{\mathcal{T}}\left[\lambda_{t \omega}^{\mathrm{D}} P_{t \omega}^{\mathrm{D}, w p p}+\lambda_{t \omega}^{\mathrm{A}} P_{t \omega}^{\mathrm{A}, w p p}+\lambda_{t \omega}^{\mathrm{D}}\left(r_{t \omega}^{+} \Delta_{t \omega}^{+, w p p}-r_{t \omega}^{-} \Delta_{t \omega}^{-, w p p}\right)\right]+\beta\left(\zeta-\frac{1}{1-\alpha} \sum_{\Omega} \pi_{\omega} \xi_{\omega}\right)$, 


$$
\begin{aligned}
\text { s.t. } & \\
\forall t, \omega: & P_{t \omega}^{w p p}=P_{t \omega}^{\mathrm{D}, w p p}+P_{t \omega}^{\mathrm{A}, w p p}, \\
\forall t, \omega: & P_{t \omega}^{w p p} \leq P^{\mathrm{W}, \max }, \\
\forall t, \omega: & P_{t \omega}^{\mathrm{D}, w p p} \leq P^{\mathrm{W}, \mathrm{max}}, \\
\forall t, \omega: & \Delta_{t \omega}^{w p p}=P_{t \omega}^{\mathrm{W}}-P_{t \omega}^{w p p}, \\
\forall t, \omega: & \Delta_{t \omega}^{w p p}=\Delta_{t \omega}^{+, w p p}-\Delta_{t \omega}^{-, w p p}, \\
\forall t, \omega: & \Delta_{t \omega}^{+, w p p} \leq P_{t \omega}^{\mathrm{W}}, \\
\forall t, \omega: & \Delta_{t \omega}^{-, w p p} \leq P^{\mathrm{W}, \max }, \\
\forall t, \omega, \omega^{\prime}: & \left(P_{t \omega}^{\mathrm{D}, w p p}-P_{t \omega^{\prime}}^{\mathrm{D}, w p p}\right) \cdot\left(\lambda_{t \omega}^{\mathrm{D}}-\lambda_{t \omega^{\prime}}^{\mathrm{D}}\right) \geq 0, \\
\forall t, \omega, \omega^{\prime}: & P_{t \omega}^{\mathrm{D}, w p p}=P_{t \omega^{\prime}}^{\mathrm{D}, w p}: \lambda_{t \omega}^{\mathrm{D}}=\lambda_{t \omega^{\prime}}^{\mathrm{D}}, \\
\forall t, \omega, \omega^{\prime}: & P_{t \omega}^{\mathrm{A}, w p p}=P_{t \omega^{\prime}}^{\mathrm{A}, w p}: \lambda_{t \omega}^{\mathrm{A}}=\lambda_{t \omega^{\prime}}^{\mathrm{A}}, \\
\forall t, \omega: & -\ell \cdot P_{t \omega}^{\mathrm{D}, w p p} \leq P_{t \omega}^{\mathrm{A}, w p p} \leq \ell \cdot P_{t \omega}^{\mathrm{D}, w p p}, \\
\forall \omega: & \zeta-\sum_{\mathcal{T}}\left[\lambda_{t \omega}^{\mathrm{D}} P_{t \omega}^{\mathrm{D}, w p p}+\lambda_{t \omega}^{\mathrm{A}} P_{t \omega}^{\mathrm{A}, w p p}+\lambda_{t \omega}^{\mathrm{D}}\left(r_{t \omega}^{+} \Delta_{t \omega}^{+, w p p}-r_{t \omega}^{-} \Delta_{t \omega}^{-, w p p}\right)\right] \leq \xi_{\omega}, \\
\forall \omega: & \xi_{\omega} \geq 0, \\
\forall t, \omega: & P_{t \omega}^{w p p}, P_{t \omega}^{\mathrm{D}, w p p}, \Delta_{t \omega}^{+, w p p}, \Delta_{t \omega}^{-, w p p} \geq 0 .
\end{aligned}
$$

The optimization (35) to be maximized shows the optimization problem for WPP when it acts independently in the three settlement market. The total power that can be offered by WPP is bounded by the maximum capacity of WPP generation, $P^{\mathrm{W}, \max }$ is as shown in Constraints (37). Accordingly, the upper limit of DA offered by WPP is $P^{\mathrm{W}, \max }$ as can be seen in (38). Constraints (39) demonstrate the total deviation, which is the sum of generated power minus the total offered power. Constraints (41) and (42) present the maximum possible amount of positive and negative power deviations, respectively. The maximum positive deviation is equal to the amount of generated wind power. The maximum negative deviation is equal to the wind farm capacity. The positive variables are specified by (48)-(49).

DRA meets the requirements of its clients and participates in a two-settlement market [22]. It offers the energy in the batteries of EVs to the markets in order to mitigate the costs. The resulted formulation is shown by (50):

Max. $\quad \mathcal{E}^{\mathrm{DRP}}=\sum_{\Omega} \pi_{\omega} \sum_{\mathcal{T}}\left[\lambda_{t \omega}^{\mathrm{D}}\left(L_{t \omega}^{\mathrm{D}}-D_{t \omega}^{\mathrm{D}}\right)+\lambda_{t \omega}^{\mathrm{A}}\left(L_{t \omega}^{\mathrm{A}}-D_{t \omega}^{\mathrm{A}}\right)-\Pi_{t \omega}\right]+\beta\left(\zeta-\frac{1}{1-\alpha} \sum_{\Omega} \pi_{\omega} \xi_{\omega}\right)$,

s.t.

(5)-(11),

(24)-(28),

$$
\begin{aligned}
\forall \omega: & \zeta-\sum_{\mathcal{T}}\left[\lambda_{t \omega}^{\mathrm{D}}\left(L_{t \omega}^{\mathrm{D}}-D_{t \omega}^{\mathrm{D}}\right)+\lambda_{t \omega}^{\mathrm{A}}\left(L_{t \omega}^{\mathrm{A}}-D_{t \omega}^{\mathrm{A}}\right)-\Pi_{t \omega}\right] \leq \xi_{\omega}, \\
\forall \omega: & \xi_{\omega} \geq 0 \\
\forall t, \omega: & L_{t \omega}, L_{t \omega}^{\mathrm{D}}, L_{t \omega}^{\mathrm{A}}, D_{t \omega}^{\mathrm{D}}, D_{t \omega}^{\mathrm{A}} \geq 0 \\
\forall t, \omega, v: & \varrho_{t \omega v}^{+}, \varrho_{t \omega v}^{-}, \rho_{t \omega v}, \varrho_{t \omega \nu}^{S O C} \geq 0, \\
\forall t, \omega, v: & v_{t \omega v}, \theta_{t \omega v} \in\{0,1\} .
\end{aligned}
$$

Equation (50) is the optimization problem that DRA faces. The objective function consists of the revenues that it earns from offering to the DA and adjustment markets minus the cost that it incurs for buying energy from these markets. It is assumed that DRA does not participate in the balancing market. 
Table 1: Specifications of ARMA Models

\begin{tabular}{lccc}
\hline & Day-ahead & Adjustment & Balancing \\
\hline ARLags & 1 & 1 & 1 \\
SARLags & 24 & 24 & 24 \\
MALags & 1,2 & 1 & 0 \\
SMALags & 24,168 & 0 & 0 \\
\hline
\end{tabular}

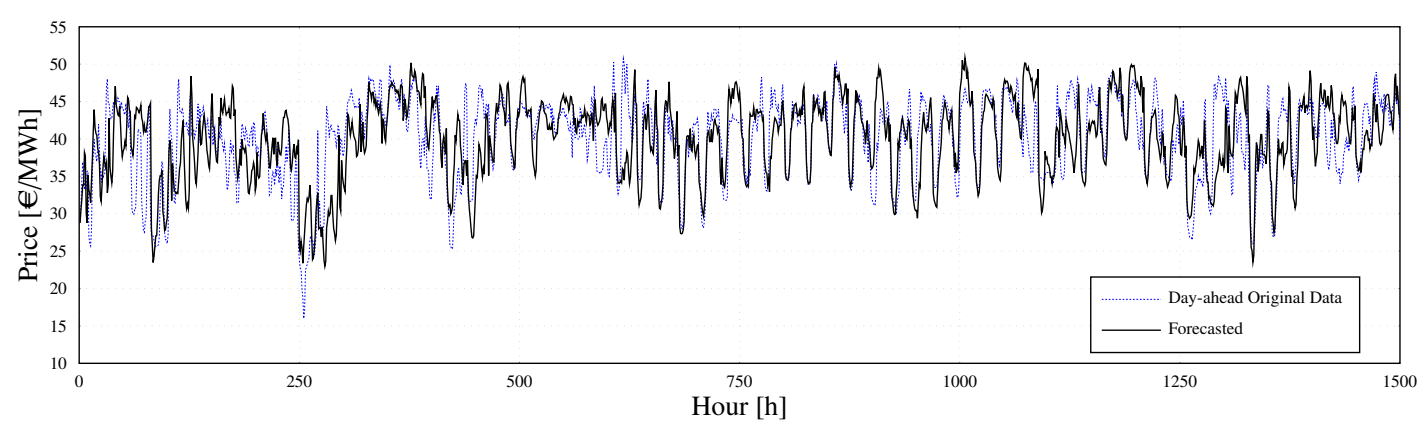

Figure 4: Day-ahead price forecast

\section{Case Study}

In order to assess the efficiency of the proposed scheme, a detailed illustrative case study is presented in this section. The case study is implemented using the data of day-ahead, intraday (adjustment), and balancing markets of Iberian electricity market [49]. The wind farm data is extracted from the data of Sotavento wind farm, which is publicly available [50]. In this case study, an EV fleet with the total number of $1000 \mathrm{EVs}$ consisting of three types of EVs are considered. In the following, firstly, the input data are explained, and afterwards the evaluation results are presented.

\subsection{Data}

This part explains the modelling of four stochastic processes, namely, day-ahead price, adjustment price, imbalance coefficients, and wind farm production. Also the parameters of EVs are explained in detail.

\subsubsection{Market Prices}

Based on (1), the ARMA parameters for the three markets are derived and presented in Table 1. 100 scenarios per hour are generated for each of the market prices. Fig. 4 compares the results of ARMA forecast with the real prices of DA market. The derived forecast curve is the average of 100 scenarios for the price of each hour. Variance is generated using the Gaussian Distributed Conditional Heteroskedasticity $(\mathrm{GARCH})$ model in the MATLAB software.

Table 2: Mixture of Four von Mises Distributions

\begin{tabular}{cccc} 
& \multicolumn{3}{c}{ Table 2: Mixture of Four von Mises Distributions } \\
\hline & $\mu$ & $\kappa$ & Component Proportion \\
\hline 1 & +1.5376 & 03.8664 & 0.4115 \\
2 & -0.3139 & 01.5712 & 0.3229 \\
3 & -2.4821 & 07.5677 & 0.1183 \\
4 & +2.5633 & 17.2077 & 0.1473 \\
\hline
\end{tabular}


Table 3: EVs Technical Data. Charging efficiencies are calculated based on the work of Ref. [48]

\begin{tabular}{lccc}
\hline Battry features & BMW i3 & Nissan Leaf & Kia Soul EV \\
\hline Capacity [kWh] & 33 & 40 & 30 \\
Full Charging Time [h] & 4.8 & 7.5 & 5 \\
Charging Efficiency [\%] & 93 & 81 & 91 \\
Charge/Discharge Rate [kW] & 7.4 & 6.6 & 6.6 \\
\hline
\end{tabular}
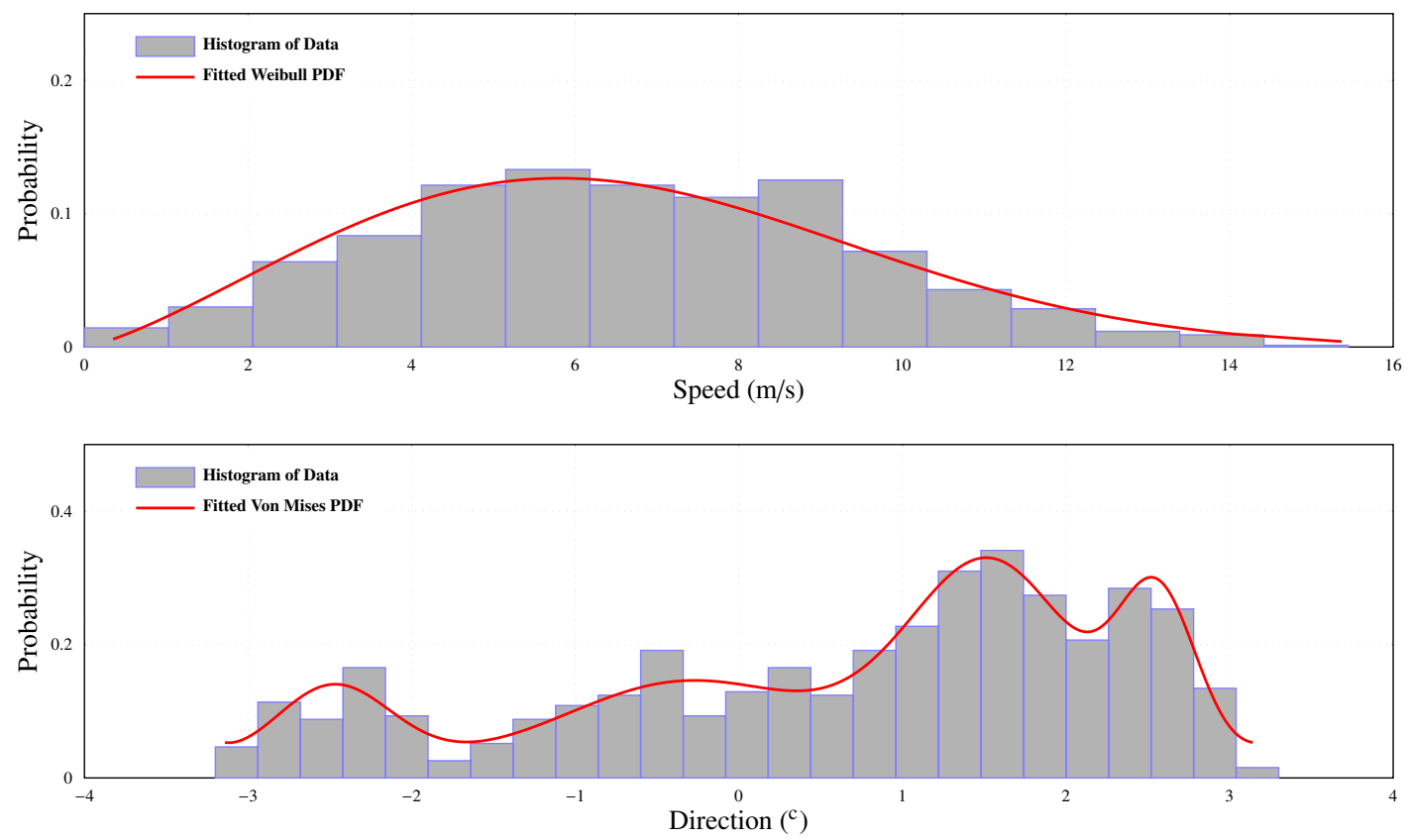

Figure 5: Fitted probability density functions (PDFs) for wind speed and direction. (Using these functions, scenarios for ANFIS inputs are generated.)

\subsubsection{Wind Power Generation}

In order to model the wind farm production, proper distributions for the speed and direction of wind are needed. Moreover, the ANFIS parameters should be decided [44]. As mentioned in Section 2.3, a fuzzy inference system is used to model the wind farm operation. The inputs of this fuzzy system are the wind speed and direction which are generated using Weibull distribution and a mixture of four von Mises distributions, respectively. Parameters of the Weibull distribution are $k=2.2929$ and $\lambda=7.4626$ [51, 52]. Parameters of the four von Mises distributions used to generate scenarios for wind direction are presented in Table 2 [53-55]. Fig. 5 depicts the fitted distributions for the wind speed and direction, along with their normalized histograms.

The number of generated scenarios for WPP production at each hour is considered to be 100 too. Fig. 6 shows the forecasted generation obtained by the fuzzy inference system and the real generation of WPP.

As the initial number of scenarios is very high, scenario reduction methods should be applied on the set of scenarios. In this respect, a scenario reduction method using a forward selection algorithm [56] is applied to the previously generated scenarios. The numbers of reduced scenarios for DA, adjustment, and balancing prices and WPP production are $5,10,5$, and 10 , respectively. Therefore, the three-stage optimization problem will have $5 \times 10 \times 5 \times 10=2500$ scenarios. 


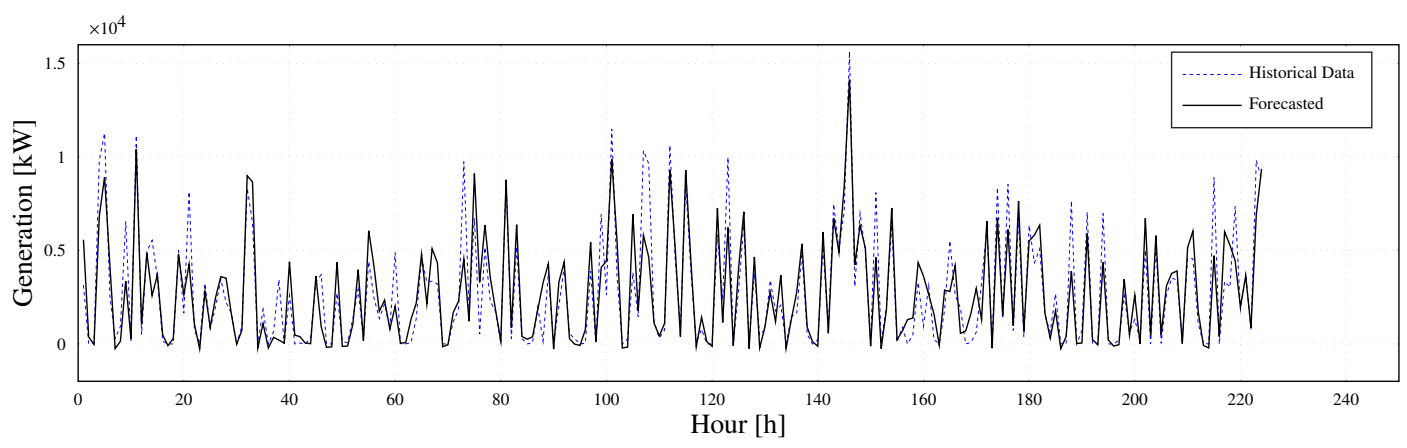

Figure 6: Wind farm generation forecast

Table 4: Expected Profit for Test Days

\begin{tabular}{|c|c|c|c|c|c|c|c|}
\hline \multicolumn{3}{|c|}{ Case } & $01 / 02 / 2017$ & $02 / 02 / 2017$ & $03 / 02 / 2017$ & $04 / 02 / 2017$ & $05 / 02 / 2017$ \\
\hline \multirow{4}{*}{ 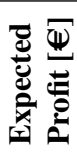 } & \multirow{3}{*}{ DO* } & WPP & 1724.5 & 2654.5 & 1514.0 & 2669.9 & 2630.3 \\
\hline & & DRA & 523.4 & 439.7 & 357.0 & 413.7 & 336.8 \\
\hline & & Sum & 2247.9 & 3094.2 & 1871.0 & 3083.6 & 2967.1 \\
\hline & $\mathbf{J O}^{* *}$ & VPP & 2693.9 & 3191.7 & 2081.5 & 3434.2 & 3767.8 \\
\hline
\end{tabular}

${ }^{*}$ Disjoint Operation $\quad$ ** Joint Operation

\subsubsection{EV Parameters}

Three different types of EVs from three manufacturers are considered in this study consisting of 300 Nissan Leaf $^{1}$, 300 Kia Soul EV $V^{2}$, and $400 B M W i 3^{3}$. The technical information of these EVs is shown in Table 3.

In the next step, three different driving patterns are modeled for each category of vehicles in order to investigate the effects of driving patterns on DRA's decision making and profits. Driving pattern of an EV is reflected by its average electricity usage (average loss of charge). Therefore, as seen in Fig. 7, three driving patterns representing low, medium, and high usage are considered for each EV category. These values are random variables which are generated by a normal distribution function.

In this figure, the average time that EVs are driving hence, not accessible for charging or discharging, is around 8.6 percent. As it was noted earlier, most of the vehicles are in the parking status more than 95 percent of the time.

Average Driving Time Percentage: $8.625 \%$

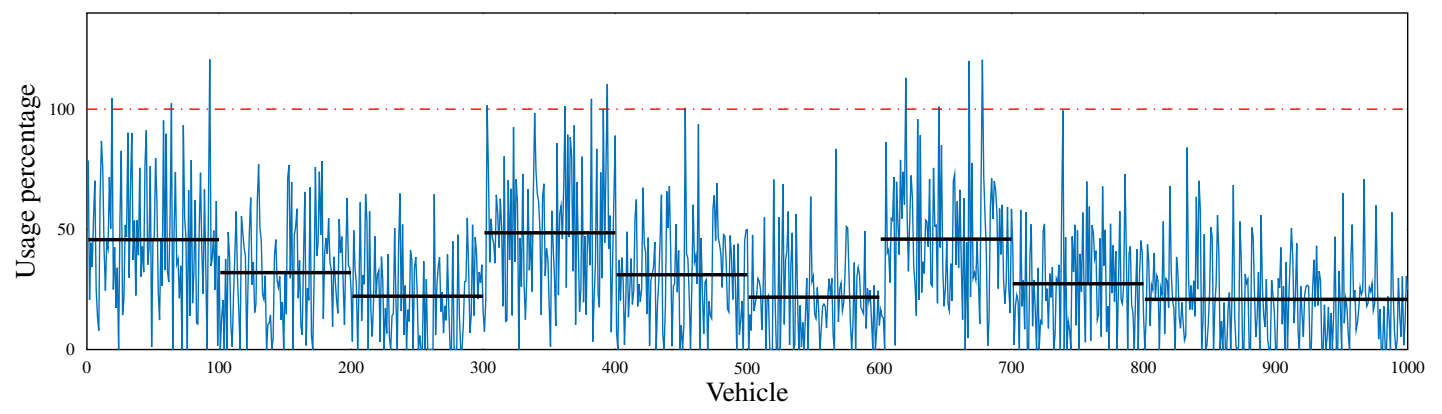

Figure 7: Different consumption types

\footnotetext{
${ }^{1}$ https://www.nissanusa.com/content/dam/Nissan/us/vehicle-brochures/2018/2018-leaf-brochure-en.pdf

${ }^{2}$ https://www.kia.com/us/k3/content/media/all/vehicles/soul-ev/brochures/brochure_soul-ev_2018.pdf

${ }^{3}$ https://www.bmwusa.com/vehicles/bmwi/bmw-i3/bmw-i3-features-and-specs/specifications.html
} 
Table 5: Movement Information of EV Fleet

\begin{tabular}{|c|c|c|c|c|c|c|c|c|c|c|c|c|c|c|c|c|c|c|c|c|c|}
\hline \multirow{3}{*}{$\begin{array}{c}\text { Case } \\
\text { Vehicle }\end{array}$} & \multicolumn{9}{|c|}{ Pattern1 } & \multicolumn{9}{|c|}{ Pattern2 } & \multirow{2}{*}{\multicolumn{3}{|c|}{$\begin{array}{c}\text { Pattern3 } \\
\text { Type1 }\end{array}$}} \\
\hline & \multicolumn{3}{|c|}{ Type1 } & \multicolumn{3}{|c|}{ Type2 } & \multicolumn{3}{|c|}{ Type3 } & \multicolumn{3}{|c|}{ Type1 } & \multicolumn{3}{|c|}{ Type2 } & \multicolumn{3}{|c|}{ Type3 } & & & \\
\hline & $\mathrm{N}^{*}$ & $\mathrm{~K}^{* *}$ & $\mathrm{~B}^{\dagger}$ & $\mathrm{N}$ & $\mathrm{K}$ & B & $\mathrm{N}$ & $\mathrm{K}$ & B & $\mathrm{N}$ & $\mathrm{K}$ & B & $\mathrm{N}$ & $\mathrm{K}$ & B & $\mathrm{N}$ & K & B & $\mathrm{N}$ & $\mathrm{K}$ & B \\
\hline $\begin{array}{l}\text { Movement } \\
\text { Percent- } \\
\text { age }\end{array}$ & 9 & 10.5 & 5.8 & 7.7 & 11.9 & 6.1 & 9.8 & 10.8 & 5.5 & 15.2 & 21 & 10.3 & 15.4 & 21.7 & 10.9 & 16.6 & 21.5 & 9.9 & 9 & 8.2 & 9.2 \\
\hline $\begin{array}{c}\text { SOC } \\
\text { loss }\end{array}$ & 1 & 1.2 & 0.6 & 0.8 & 1.3 & 0.7 & 0.7 & 0.9 & 0.4 & 1.1 & 1.5 & 0.7 & 1 & 1.6 & 0.8 & 0.9 & 1.1 & 0.5 & 1.1 & 0.8 & 0.9 \\
\hline
\end{tabular}

${ }^{*} \mathrm{~N}$ : Nissan Leaf $\quad{ }^{* *} \mathrm{~K}$ : Kia Soul EV $\quad{ }^{\dagger} \mathrm{B}: \mathrm{BMW}$ i3

The 7 different pattern-types can be considered as 7 different case studies, in which, each pattern has different "average driving time". For instance, the average driving time of Pattern 1 is $8.6 \%$, but the average driving time of Pattern 2 is over $15 \%$ (Fig. $7 \&$ Table 5). It means that vehicles are unavailable for the network in $8.6 \%$ of their time, in Pattern 1. In other words, the average time that vehicles are at network's disposal is $((100-8.6)) / 100 \times 24 \approx 21.9$ h. Whereas, the average available time is $((100-15)) / 100 \times 24 \approx 20.4 \mathrm{~h}$, in Pattern 2 . Accordingly, the network can enjoy more of EVs' storage capability, in Pattern 1.

Moreover, each pattern is consisted of several types (Table 5). Every type represents different kind of practical usage of EVs. For instance, it is assumed that EVs could move 24/7 in Type 1, while, in Type 2, the assumption is that they are parked between 00:00 - 06:00 am. Type 3 assumes not only are vehicles parked during 00:00 - 06:00 am, but also they are parked from 09:00 am to 02:00 pm. A Type 1 EV could be an ambulance or a fire engine. On the other hand, a Type $2 \mathrm{EV}$ could be a taxi that its owner sleeps between 00:00 - 06:00 am, but possibly uses his vehicle in any other hour. Finally, a Type $3 \mathrm{EV}$ is owned by a regular user who rests at night and is in his workplace during 09:00 am - 02:00 pm, hence his EV is parked.

For the first 6 patterns, the batteries of EVs are assumed to be half charged at the beginning of time horizon, while for the seventh pattern they are completely discharged.

EV parameters are determined according to Table 3. EV initial SOC is equal to the minimum battery SOC.

Parameters $\rho_{t \omega v}$ are determined by EV owners and are assumed to be given. The EV owners inform the DRA the time slots of tomorrow at which their vehicles are needed for driving. If the vehicle is not needed at the time slot $t, \rho_{t \omega v}=0$, otherwise, $\rho_{t \omega v} \neq 0$. The value of $\rho_{t \omega v}$ should be known for $\rho_{t \omega v} \neq 0$, allowing the DRA to schedule it. The mechanism for determining the value of $\rho_{t \omega v}$ is beyond the scope of this paper. Here it is assumed that $\rho_{t \omega v}$ are known parameters which are randomly simulated using some normal distribution. Obviously, values of $\rho_{t \omega v}$ must satisfy EVs' technical constraints. The other assumption considered in this case study is that $\rho_{t \omega v}=0$ for $t \leq 6$. This assumption is made because the first six hours are off-peak periods and vehicles most likely are not needed. It should be noted that this is an optional assumption and does not affect the simulation procedure.

\subsection{Simulation and Results}

The following model parameters are used in the simulations:

$$
\left[P^{\mathrm{W}, \max }, \alpha, \ell, \eta, \beta\right]=[17.56,0.95,0.3,1,0.5] .
$$

\subsubsection{Energy Offers and Expected Profits}

Fig. 8 shows the DRA's energy purchase (battery charge) and DRA's energy sell (battery discharge) along with the total energy used due to the driving, in each time period. The battery usages in driving and V2G modes are shown as positive values as it benefits the vehicle's owners or DRA, while the vehicles' charging during the G2V mode is negative since DRA incurs costs for purchasing energy from the market.

As it was mentioned in the preceding sections, the initial and final battery SOC are assumed to be equal. Therefore, if other types of energy loss are neglected, the total amount of charged energy of an EV will be equal to the EV's discharged energy due to the driving and selling energy to the grid.

This figure also depicts the DA market prices for the considered time period. It can be seen that DRA purchases most of its needed energy when the market prices are low (off-peak hours) and sells the energy during those time 


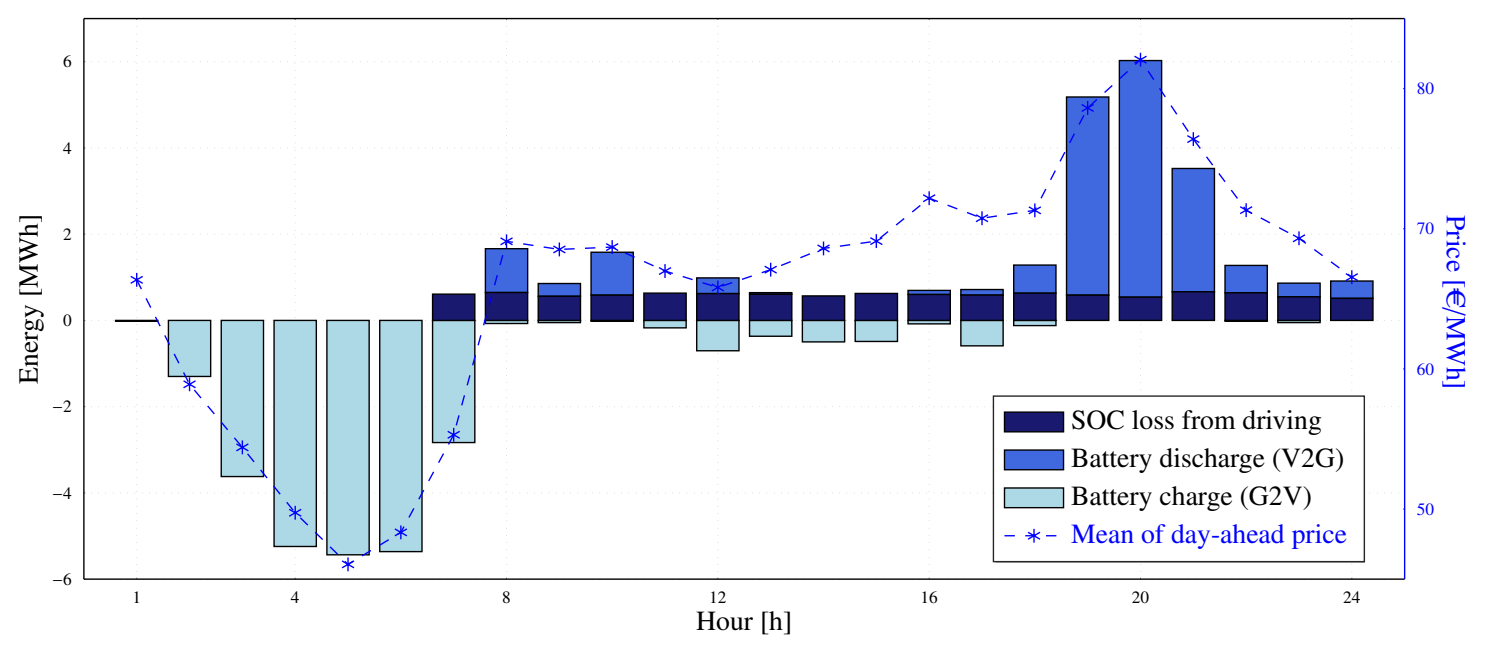

Figure 8: DRA energy purchase, sell, and consumption per hour (05/02/2017)

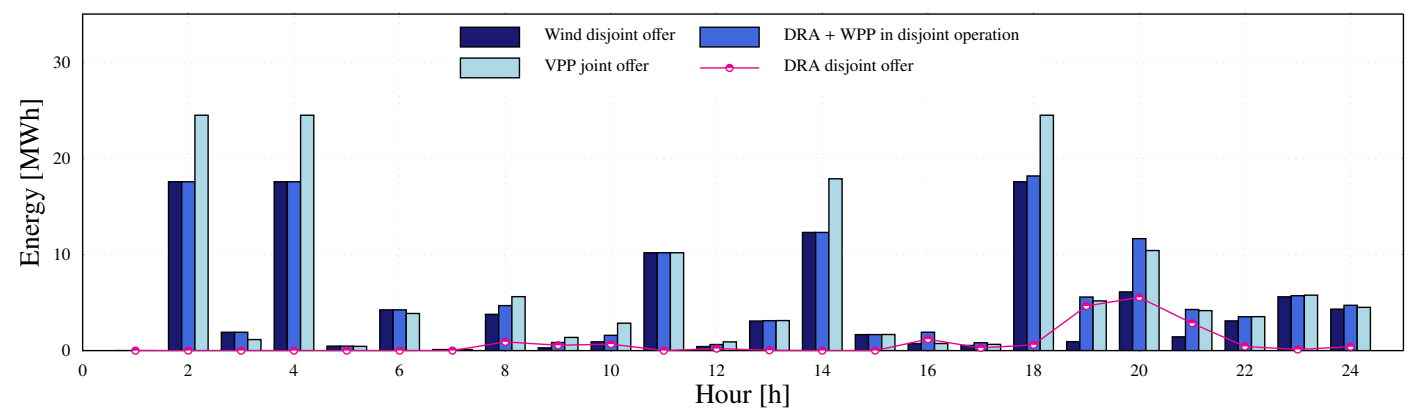

Figure 9: Total energy offer in two configurations $(05 / 02 / 2017)$

periods with the highest prices. DRA does not purchase any electricity in time periods \#19, \#20, and \#24, while it does not sell any electricity in time periods \#1 to \#7, \#14, and \#15.

Fig. 9 shows the energy quantity offered to the market by WPP and DRA in the disjoint operation and by VPP in the joint operation, respectively. As demonstrated, in most time periods, the VPP offer is higher than the sum of WPP and DRA offers in the disjoint operation.

Table 4 represents the expected profit of different configurations during a five-day period. Based on these results, the expected profit of VPP under the joint operation is higher in comparison with the independent operation, since DRA can mitigate the WPP's uncertainty, store the excess generation during the off-peak hours, and sell the energy to the grid in the peak-hours.

Also, the hourly expected profit in each time period obtained for these two configurations is shown in Fig. 10.

\subsubsection{Effect of Driving Patterns}

Fig. 11 compares the operation of vehicles for 7 pattern-type cases which are represented in Table 5. EVs' ability in storing the electricity and selling it back to the grid can benefit EV owners. So, to emphasize this ability, the operation in each case is also compared for two different configurations: 1) when the EVs buy and sell electricity to the market (the case with discharging) and, 2) when the EVs solely buy electricity from the grid (the case without discharging). In the first configuration, EVs need to buy more electricity from the grid. However, they can benefit from this purchase as they sell a part of this procured electricity to the market. Also, in this configuration, EVs' benefit is investigated with or without considering battery degradation cost.

In summary, for comparison purposes, the problem is solved when the battery discharge (V2G) is considered or not, in each case. Also, when considering the discharge, the results are further investigated with/without battery 


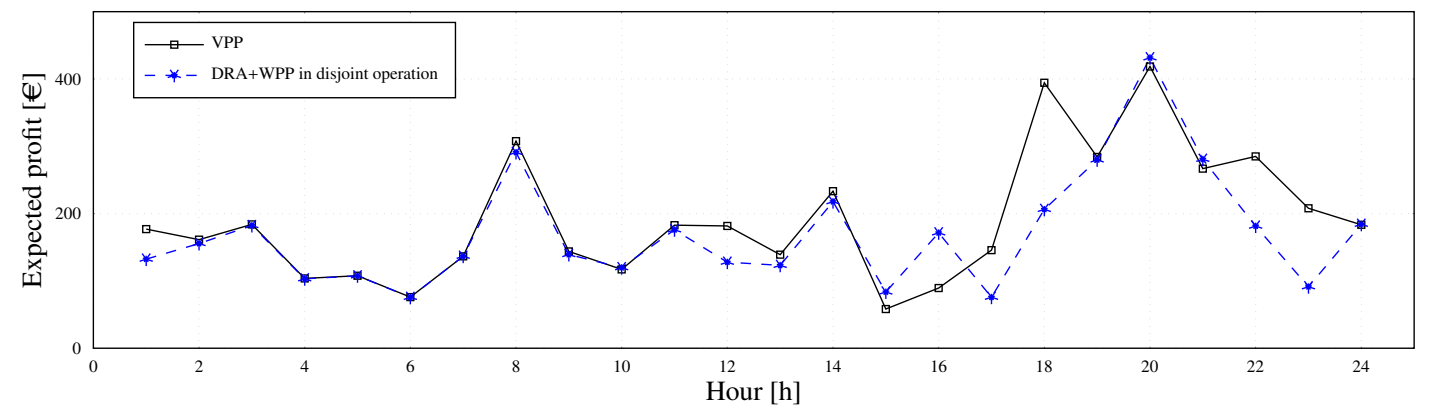

Figure 10: Hourly profit for joint and disjoint operations (05/02/2017)

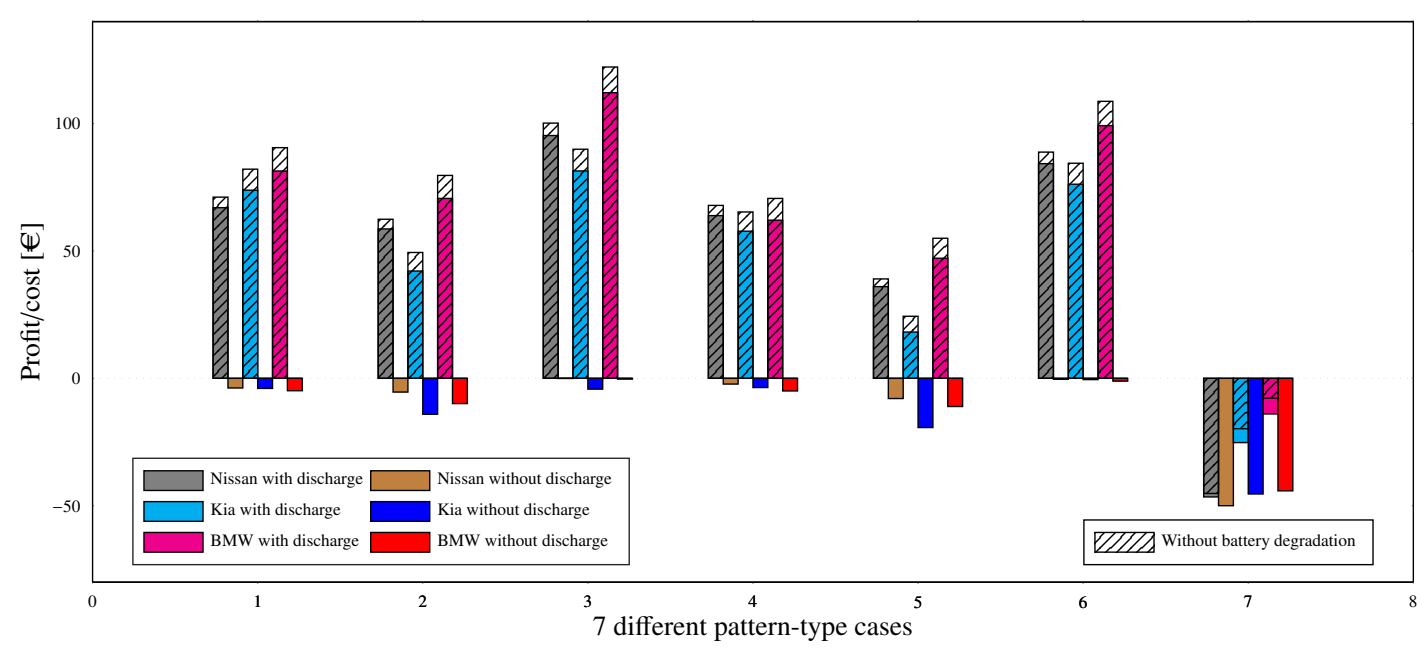

Figure 11: The profit/cost for different driving patterns $(05 / 02 / 2017)$ 


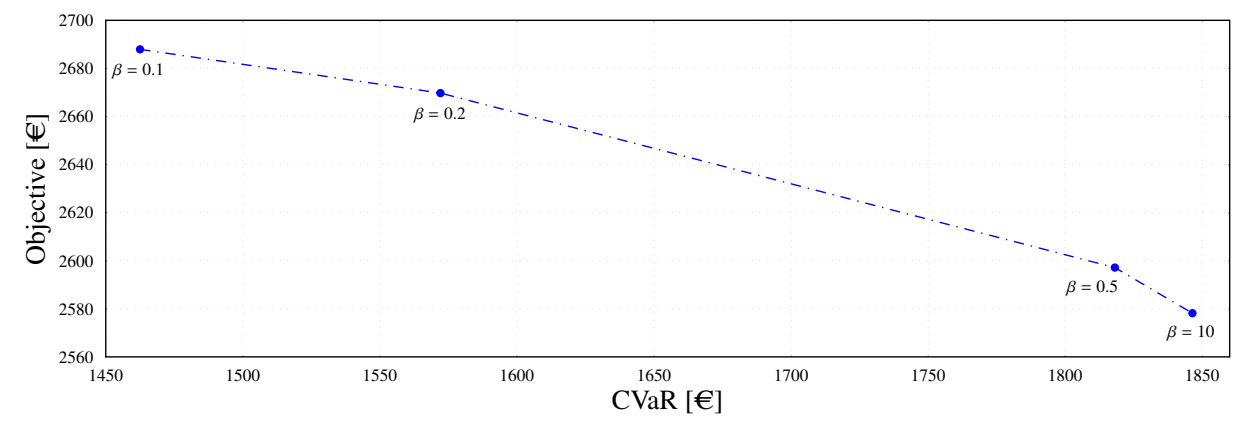

Figure 12: Values of objective function for different values of parameter $\beta$

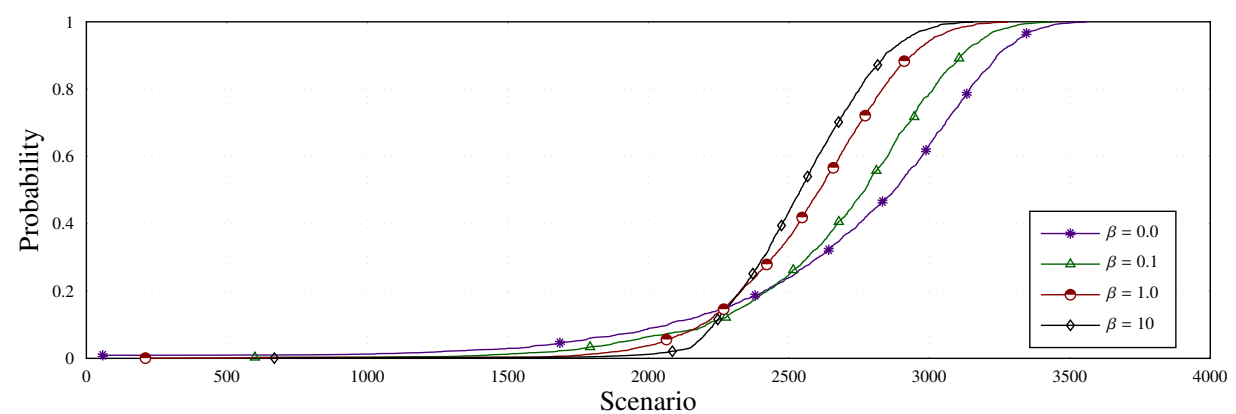

Figure 13: Probability of occurrence of scenarios for different values of $\beta$

degradation.

$$
\text { Each case }\left\{\begin{array}{l}
\text { Considering V2G }\left\{\begin{array}{l}
\text { Battery degradation is considered } \\
\text { Battery degradation is not considered }
\end{array}\right. \\
\text { Without considering V2G (without battery degradation) }
\end{array}\right.
$$

The results show that in all the cases EVs benefit from trading in the electricity market. Here, the profit/cost is calculated as the total amount of revenues minus the total amount of costs. For the first 6 patterns, EVs can even have some profits. For the pattern 7, as the initial SOC is zero, the EVs need to buy more energy and they cannot earn profits.

\subsubsection{Impact of Risk on the Problem}

The previous results were obtained by considering the parameter $\beta$ to be 0.5 . Here, to investigate the effect of risk on energy transactions as well as profits, $\beta$ is changed from 0 to 10 .

As it can be seen from Fig. 12, the value of CVaR increases about 400 Euros by increasing $\beta$ from 0 to 10 . This increase in CVaR leads to a decrease of around 100 Euros in the expected profits.

Fig. 13 depicts the cumulative distribution function (CDF) of profit. For the probabilities between 0.1 and 0.9 , the range of variations in the expected profit is limited to 600 for $\beta=10$, while it is 1300 for $\beta=0$. It is expected as for the higher values of $\beta$, the probability of occurrence of those scenarios with low profits is almost zero.

Comparison of these two figures demonstrates the advantage of adding risk measure to the problem. By increasing the CVaR the VPP faces a low decrease in its expected profit. On the other hand, the risk of experiencing low profit is diminished very well.

The VPP offers to the DA market, for different values of $\beta$, is depicted in Fig. 14. According to these results, the offers will decrease by increasing the value of $\beta$. 


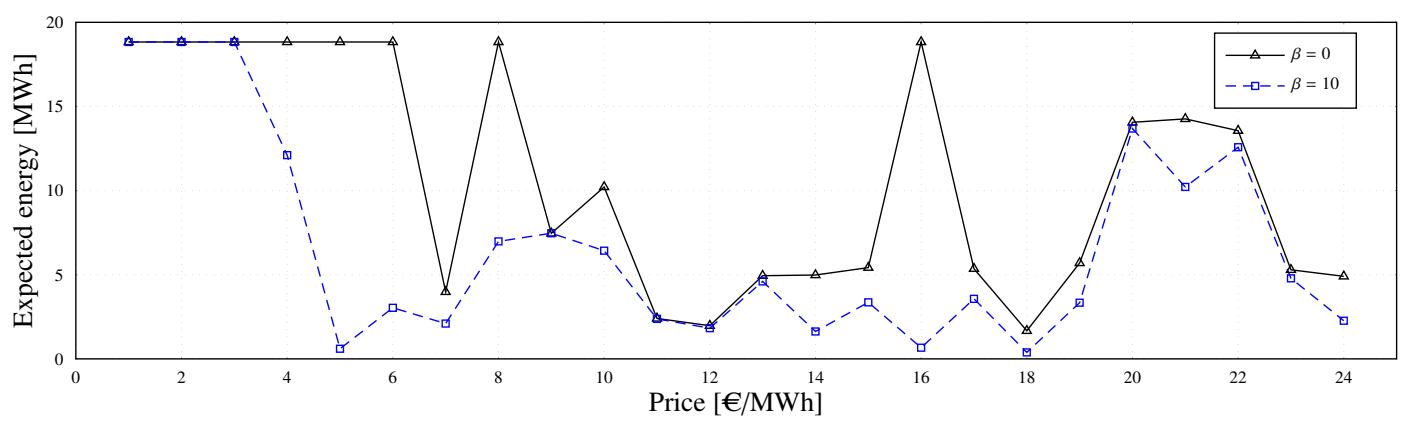

Figure 14: DA market offer for different values of $\beta$

\section{Conclusion}

Since the number of electric vehicles is increasing rapidly and they do not require initial investment from grid operators or power suppliers, they can be seen as potential flexible storages to facilitate the integration of renewable resources. In this regard, this paper formulated a three-stage mixed-integer stochastic programming problem for a virtual power plant, consisting of a wind power producer and a demand response aggregator with electric vehicle clients, which participate in a pool-based market. The paper takes into account the detailed characteristics of each vehicle, battery degradation, and uncertainties.

Three measures are taken in order to obtain the results which are as close as possible to the real case scenarios. Firstly, the real data of three different electric vehicles from three main manufacturers were used. Secondly, the number of vehicles considered is chosen to be sufficiently large so as to investigate its impact on the producers decision making and market interactions. This is a feasible assumption since the number of electric vehicles in the networks is increasing steadily and aggregation of 1000 vehicles will be achieved easily in the near future. Thirdly, the various pattern-type cases are defined to simulate the actual behavior of vehicle owners.

Numerical studies were performed to study three main aspects. Market prices and wind generation were forecasted through ARMA models and an ANFIS system, respectively, based on historical data of real wind power generators and Spanish electricity market. As the main goal, the paper investigates the disjoint interaction of the wind producer and the aggregator, and compares the results with the joint operation case. Based on numerical simulations, it was observed that the joint operation as a virtual power plant has significant benefits for vehicle owners as well as producer, and it outperforms the independent operation. The effect of different driving patterns on the offers and profits/costs was studied, where 7 cases were defined which mimic various driving patterns. Finally, the impact of involving the risk measure into the formulation was also shown.

\section{References}

[1] M. Zugno, J. M. Morales, P. Pinson, H. Madsen, Pool strategy of a price-maker wind power producer, IEEE Transactions on Power Systems 28 (3) (2013) 3440-3450.

[2] L. Baringo, A. J. Conejo, Offering strategy of wind-power producer: A multi-stage risk-constrained approach, IEEE Transactions on Power Systems 31 (2) (2016) 1420-1429.

[3] H. Zhao, Q. Wu, S. Hu, H. Xu, C. N. Rasmussen, Review of energy storage system for wind power integration support, Applied Energy 137 (Supplement C) (2015) 545-553.

URL http://www.sciencedirect.com/science/article/pii/S0306261914004668

[4] M. Khalid, R. P. Aguilera, A. V. Savkin, V. G. Agelidis, On maximizing profit of wind-battery supported power station based on wind power and energy price forecasting, Applied Energy 211 (2018) 764-773.

[5] L. Ju, H. Li, J. Zhao, K. Chen, Q. Tan, Z. Tan, Multi-objective stochastic scheduling optimization model for connecting a virtual power plant to wind-photovoltaic-electric vehicles considering uncertainties and demand response, Energy Conversion and Management 128 (2016) $160-177$.

[6] M. G. Vayá, G. Andersson, Self scheduling of plug-in electric vehicle aggregator to provide balancing services for wind power, IEEE Transactions on Sustainable Energy 7 (2) (2016) 886-899.

[7] A. K. Varkani, A. Daraeepour, H. Monsef, A new self-scheduling strategy for integrated operation of wind and pumped-storage power plants in power markets, Applied Energy 88 (12) (2011) 5002-5012. 
[8] M. S. Al-Swaiti, A. T. Al-Awami, M. W. Khalid, Co-optimized trading of wind-thermal-pumped storage system in energy and regulation markets, Energy 138 (2017) 991-1005.

[9] J. Aghaei, M. Barani, M. Shafie-khah, A. A. S. de la Nieta, J. P. S. Catalão, Risk-constrained offering strategy for aggregated hybrid power plant including wind power producer and demand response provider, IEEE Transactions on Sustainable Energy 7 (2) (2016) 513-525.

[10] J. Katz, F. M. Andersen, P. E. Morthorst, Load-shift incentives for household demand response: Evaluation of hourly dynamic pricing and rebate schemes in a wind-based electricity system, Energy 115 (2016) 1602-1616.

[11] H. M. I. Pousinho, V. M. F. Mendes, J. P. d. S. Catalão, A stochastic programming approach for the development of offering strategies for a wind power producer, Electric Power Systems Research 89 (2012) 45-53.

[12] D. R. Melo, A. Trippe, H. B. Gooi, T. Massier, Robust electric vehicle aggregation for ancillary service provision considering battery aging, IEEE Transactions on Smart Grid.

[13] T. Sousa, H. Morais, J. Soares, Z. Vale, Day-ahead resource scheduling in smart grids considering vehicle-to-grid and network constraints, Applied Energy 96 (2012) 183-193.

[14] M. Asensio, J. Contreras, Risk-constrained optimal bidding strategy for pairing of wind and demand response resources, IEEE Transactions on Smart Grid 8 (1) (2017) 200-208.

[15] L. Hancher, Think topic 11: shift, not drift: Towards active demand response and beyond, http://www.eui.eu/Projects/THINK/ Documents/Thinktopic/Topic11digital.pdf (2013).

[16] T. K. Kristoffersen, K. Capion, P. Meibom, Optimal charging of electric drive vehicles in a market environment, Applied Energy 88 (5) (2011) 1940-1948.

[17] C. Peng, J. Zou, L. Lian, L. Li, An optimal dispatching strategy for v2g aggregator participating in supplementary frequency regulation considering ev driving demand and aggregators benefits, Applied Energy 190 (2017) 591-599.

[18] B. Škugor, J. Deur, A bi-level optimisation framework for electric vehicle fleet charging management, Applied energy 184 (2016) $1332-1342$.

[19] M. A. Ortega-Vazquez, F. Bouffard, V. Silva, Electric vehicle aggregator/system operator coordination for charging scheduling and services procurement, IEEE Transactions on Power Systems 28 (2) (2013) 1806-1815.

[20] A. Perez-Diaz, E. Gerding, F. McGroarty, Coordination and payment mechanisms for electric vehicle aggregators, Applied Energy 212 (2018) $185-195$.

[21] S. Vandael, B. Claessens, M. Hommelberg, T. Holvoet, G. Deconinck, A scalable three-step approach for demand side management of plug-in hybrid vehicles, IEEE Transactions on Smart Grid 4 (2) (2013) 720-728.

[22] I. Momber, A. Siddiqui, T. G. S. Román, L. Söder, Risk averse scheduling by a pev aggregator under uncertainty, IEEE Transactions on Power Systems 30 (2) (2015) 882-891.

[23] Y. Xiong, B. Wang, C.-c. Chu, R. Gadh, Vehicle grid integration for demand response with mixture user model and decentralized optimization, Applied Energy 231 (2018) 481-493.

[24] F. Ruelens, S. Vandael, W. Leterme, B. J. Claessens, M. Hommelberg, T. Holvoet, R. Belmans, Demand side management of electric vehicles with uncertainty on arrival and departure times, in: 2012 3rd IEEE PES Innovative Smart Grid Technologies Europe (ISGT Europe), 2012, pp. $1-8$.

[25] M. Pantos, Exploitation of electric-drive vehicles in electricity markets, IEEE Transactions on Power Systems 27 (2) (2012) $682-694$.

[26] L. Baringo, R. S. Amaro, A stochastic robust optimization approach for the bidding strategy of an electric vehicle aggregator, Electric Power Systems Research 146 (2017) 362-370.

[27] N. Korolko, Z. Sahinoglu, Robust optimization of ev charging schedules in unregulated electricity markets, IEEE Transactions on Smart Grid 8 (1) (2017) 149-157.

[28] S. Pelletier, O. Jabali, G. Laporte, M. Veneroni, Battery degradation and behaviour for electric vehicles: Review and numerical analyses of several models, Transportation Research Part B: Methodological 103 (2017) 158-187.

[29] B. Han, S. Lu, F. Xue, L. Jiang, X. Xu, Three-stage electric vehicle scheduling considering stakeholders economic inconsistency and battery degradation, IET Cyber-Physical Systems: Theory \& Applications 2 (3) (2017) 102-110.

[30] M. R. Sarker, Y. Dvorkin, M. A. Ortega-Vazquez, Optimal participation of an electric vehicle aggregator in day-ahead energy and reserve markets, IEEE Transactions on Power Systems 31 (5) (2016) 3506-3515.

[31] J. M. Morales, A. J. Conejo, J. Pérez-Ruiz, Short-term trading for a wind power producer, IEEE Transactions on Power Systems 25 (1) (2010) $554-564$.

[32] S. Delikaraoglou, A. Papakonstantinou, C. Ordoudis, P. Pinson, Price-maker wind power producer participating in a joint day-ahead and real-time market, in: 2015 12th International Conference on the European Energy Market (EEM), 2015, pp. 1-5.

[33] Y. Xiao, X. Wang, X. Wang, C. Dang, M. Lu, Behavior analysis of wind power producer in electricity market, Applied energy 171 (2016) $325-335$.

[34] L. Exizidis, S. J. Kazempour, P. Pinson, Z. de Greve, F. Valle, Sharing wind power forecasts in electricity markets: A numerical analysis, Applied Energy 176 (2016) $65-73$. URL http://www.sciencedirect.com/science/article/pii/S0306261916306468

[35] A. Tavakoli, M. Negnevitsky, D. T. Nguyen, K. M. Muttaqi, Energy exchange between electric vehicle load and wind generating utilities, IEEE Transactions on Power Systems 31 (2) (2016) 1248-1258.

[36] M. E. Khodayar, L. Wu, M. Shahidehpour, Hourly coordination of electric vehicle operation and volatile wind power generation in scuc, IEEE Transactions on Smart Grid 3 (3) (2012) 1271-1279.

[37] J. Zhao, F. Wen, Z. Y. Dong, Y. Xue, K. P. Wong, Optimal dispatch of electric vehicles and wind power using enhanced particle swarm optimization, IEEE Transactions on Industrial Informatics 8 (4) (2012) 889-899.

[38] A. Haque, A. I. Saif, P. Nguyen, S. Torbaghan, Exploration of dispatch model integrating wind generators and electric vehicles, Applied energy 183 (2016) 1441-1451.

[39] M. Wang, Y. Mu, H. Jia, J. Wu, X. Yu, Y. Qi, Active power regulation for large-scale wind farms through an efficient power plant model of electric vehicles, Applied Energy 185 (2017) 1673-1683.

[40] F. Mwasilu, J. J. Justo, E. K. Kim, T. D. Do, J. W. Jung, Electric vehicles and smart grid interaction: A review on vehicle to grid and renewable 
energy sources integration, Renewable and Sustainable Energy Reviews 34 (Supplement C) (2014) 501-516.

URL http://www.sciencedirect.com/science/article/pii/S1364032114001920

[41] J. M. Lujano-Rojas, R. Dufo-López, J. L. Bernal-Agustín, J. P. Catalão, Optimizing daily operation of battery energy storage systems under real-time pricing schemes., IEEE Trans. Smart Grid 8 (1) (2017) 316-330.

[42] P. Aliasghari, B. Mohammadi-Ivatloo, M. Alipour, M. Abapour, K. Zare, Optimal scheduling of plug-in electric vehicles and renewable micro-grid in energy and reserve markets considering demand response program, Journal of Cleaner Production 186 (2018) 293-303.

[43] A. J. Conejo, M. Carrión, J. M. Morales, Decision Making Under Uncertainty in Electricity Markets, 1st Edition, Springer US, 2010.

[44] Y. Kassa, J. H. Zhang, D. H. Zheng, D. Wei, Short term wind power prediction using anfis, in: 2016 IEEE International Conference on Power and Renewable Energy (ICPRE), 2016, pp. 388-393.

[45] L. X. Wang, A Course in Fuzzy Systems and Control, Prentice-Hall, Inc., Upper Saddle River, NJ, USA, 1997.

[46] Y. Sun, H. Yue, J. Zhang, C. Booth, Minimisation of residential energy cost considering energy storage system and ev with driving usage probabilities, IEEE Transactions on Sustainable Energy.

[47] W. Kempton, J. Tomić, Vehicle-to-grid power fundamentals: Calculating capacity and net revenue, Journal of power sources 144 (1) (2005) 268-279.

[48] F. A. Amoroso, G. Cappuccino, Advantages of efficiency-aware smart charging strategies for pevs, Energy conversion and management 54 (1) (2012) 1-6.

[49] Spanish electricity market data, https://www.esios.ree.es/en (2017)

[50] Sotavento wind farm, http://www. sotaventogalicia.com/ (2017).

[51] S. A. Akdağ, A. Dinler, A new method to estimate weibull parameters for wind energy applications, Energy Conversion and Management 50 (7) (2009) 1761-1766.

URL http://www.sciencedirect.com/science/article/pii/S0196890409000934

[52] J. Seguro, T. Lambert, Modern estimation of the parameters of the weibull wind speed distribution for wind energy analysis, Journal of Wind Engineering and Industrial Aerodynamics 85 (1) (2000) 75-84

URL http://www.sciencedirect.com/science/article/pii/S0167610599001221

[53] J. Bentley, Modelling circular data using a mixture of von mises and uniform distributions (2006).

[54] I. S. Dhillon, S. Sra, Modeling data using directional distributions (Jan 2003).

[55] L. Barabesi, Generating von mises variates by the ratio-of-uniforms method, Statistica Applicata 7 (4) (1995) 417-426.

[56] H. Heitsch, W. Römisch, A note on scenario reduction for two-stage stochastic programs, Operations Research Letters 35 (6) (2007) 731-738. URL http://www.sciencedirect.com/science/article/pii/S0167637707000089 